\title{
The First Scale-Up Production of Theranostic Nanoemulsions
}

\author{
Lu Liu, ${ }^{1}$ Christina Bagia, ${ }^{1}$ and Jelena M. Janjic ${ }^{1-3, *}$
}

\begin{abstract}
Theranostic nanomedicines are a promising new technological advancement toward personalized medicine. Although much progress has been made in pre-clinical studies, their clinical utilization is still under development. A key ingredient for successful theranostic clinical translation is pharmaceutical process design for production on a sufficient scale for clinical testing. In this study, we report, for the first time, a successful scale-up of a model theranostic nanoemulsion. Celecoxib-loaded near-infrared-labeled perfluorocarbon nanoemulsion was produced on three levels of scale (small at $54 \mathrm{~mL}$, medium at $270 \mathrm{~mL}$, and large at 1,000 mL) using microfluidization. The average size and polydispersity were not affected by the equipment used or production scale. The overall nanoemulsion stability was maintained for 90 days upon storage and was not impacted by nanoemulsion production scale or composition. Cell-based evaluations show comparable results for all nanoemulsions with no significant impact of nanoemulsion scale on cell toxicity and their pharmacological effects. This report serves as the first example of a successful scale-up of a theranostic nanoemulsion and a model for future studies on theranostic nanomedicine production and development.
\end{abstract}

Key words: drug development technologies; drug discovery; inflammation

\section{Introduction}

A growing number of nanosystems is reported in recent literature as theranostic nanomedicines. ${ }^{1}$ These nanosystems are aimed to fulfill multiple roles: image disease state and therapeutic response, provide targeted drug delivery, control drug release, and image drug delivery efficacy. ${ }^{2-4}$ Such a multifunctional nanosystem design, however, increases preparation complexity, posing significant challenges for future production on a clinical scale and quality control. Consequently, this can slow down theranostic nanomedicine clinical translation and also impact pre-clinical research in animals. This is especially of concern as theranostic nanomedicines move into biomedical research as disease pathway probes or adjuvant therapies for cancer, inflammation, and other chronic diseases. Furthermore, theranostic nanomedicines offer unique opportunities for regenerative medicine as they can be applied into clinical use as imaging-supported delivery systems or tracking devices for therapeutic cells. ${ }^{1,5}$ For all theranostic nanomedicine applications, the reproducibility of animal data is directly related to their quality. Most pre-clinical reports on theranostics do not discuss, or discuss in very limited amount of detail, how much theranostic formulation was produced for animal testing. The goal of this study is two-fold: (1) draw attention to the need for further research on scalable processes for production of theranostic nanosystems; and (2) showcase one successful example where theranostic nanoparticles were produced on scale-up to $1,000 \mathrm{~mL}$. To achieve these goals, we selected for this scale-up study one of our earlier reported theranostic nanoemulsions with celecoxib as a model poorly soluble drug. ${ }^{6}$

\footnotetext{
${ }^{1}$ Graduate School of Pharmaceutical Sciences, Mylan School of Pharmacy, and ${ }^{2}$ Chronic Pain Research Consortium, Duquesne University, Pittsburgh, Pennsylvania. ${ }^{3}$ McGowan Research Institute for Regenerative Medicine, University of Pittsburgh, Pittsburgh, Pennsylvania.

*Address correspondence to: Jelena M. Janjic, PhD, Graduate School of Pharmaceutical Sciences, Duquesne University, 415 Mellon Hall, 600 Forbes Avenue, Pittsburgh, PA 15282, E-mail: janjicj@duq.edu
}

(c) Lu Liu et al. 2015; Published by Mary Ann Liebert, Inc. This Open Access article is distributed under the terms of the Creative Commons License (http://creativecommons.org/licenses/by/4.0), which permits unrestricted use, distribution, and reproduction in any medium, provided the original work is properly credited. 
Nanoemulsions are kinetically stable emulsions with a droplet size typically between 100 and $500 \mathrm{~nm}$, high oil content, and low amounts of surfactants. ${ }^{7}$ Nanoemulsions can be applied to increase solubility and bioavailability of poorly soluble drugs ${ }^{8-12}$ used as is or incorporated into other dosage forms such as capsules and gels. ${ }^{12-14}$ They are produced by high-energy processing (microfluidization and sonication) ${ }^{15}$ and low-energy emulsification methods. ${ }^{16}$ The focus of this report is on perfluorocarbon (PFC) nanoemulsions, which are currently extensively investigated for diverse biomedical applications, such as MRI, ${ }^{5,17-19}$ ultrasound ${ }^{20}$ and photoacoustic imaging, ${ }^{21}$ oxygen delivery, ${ }^{22,23}$ and image-guided drug delivery. ${ }^{20}$ PFCs are highly biologically inert chemically stable materials that can be quantitatively detected in vivo by ${ }^{19} \mathrm{~F}$ MRI. ${ }^{18,24}$ PFC nanoemulsions are selective inflammation imaging agents. ${ }^{25-28}$ They are also an attractive platform for nanomedicine. PFC nanoemulsions can deliver the antigen to dendritic cells (DCs), boosting the immune response in DC-based vaccines, ${ }^{29}$ or deliver antiproliferative drugs. ${ }^{30}$ Although there are numerous reports on producing PFC nanoemulsions, the manufacturing process development and scale-up are not fully investigated.

In this study, we present the first drug-loaded PFC nanoemulsion (theranostic) produced on scale, quality assessments, and comparative in vitro data between small and large scales. In the previous study, we have produced, on a small scale $(25 \mathrm{~mL})$, the near infrared (NIR)-labeled perfluoropolyether (PFPE) nanoemulsion validated for stability, imaging properties, and anti-inflammatory action in vitro. ${ }^{6}$ In this study, this nanoemulsion was prepared on three levels of scale: small $(54 \mathrm{~mL})$, medium $(270 \mathrm{~mL})$, and large $(1,000 \mathrm{~mL})$. The prepared nanoemulsions were evaluated in detail in vitro for colloidal properties, stability, and in cells. Specifically, wetested nanoemulsion long-term stability upon storage for 90 days, stability when exposed to select stress tests, and evaluated for cellular toxicity and pharmacological effects in vitro. Our data demonstrate that scale-up of a celecoxib-loaded theranostic nanoemulsion is feasible and nanoemulsion quality is maintained across all scale levels tested. We hope that following this successful example of theranostic nanomedicine scale-up and manufacturing, more studies will follow leading to successful pharmaceutical development of theranostics and their clinical translation.

\section{Materials and Methods}

Materials

Pluronic ${ }^{\circledR}$ P105 was obtained from BASF Corporation. Cremophor EL (CrEL) was purchased from Sigma
Aldrich. Miglyol 812N, Dulbecco's modified Eagle's medium (DMEM), fetal bovine serum (FBS), and the Raw 264.7 cell line were purchased from ATCC. Prostaglandin $\mathrm{E}_{2}$ EIA Kit-monoclonal was purchased from Cayman Chemical Company. DiR dye was from Life Technologies. Perfluoro(polyethylene glycol dimethyl ether) or PFPE oxide $\left(\mathrm{CF}_{3} \mathrm{O}\left(\mathrm{CF}_{2} \mathrm{CF}_{2} \mathrm{O}\right)_{n} \mathrm{CF}_{3}\right.$, where $\left.n=8-13\right)$ was obtained from Exfluor Research Corporation.

\section{Equipment}

Microfluidizer M110S and Microfluidizer M-110EH-30 from Microfluidics Corporation, Westwood, Massachusetts, were used for all nanoemulsion preparations.

\section{Preparation of CrEL/P105 surfactant mixture}

The CrEL and pluronic P105 surfactant mixture was prepared as reported earlier. ${ }^{6}$ Briefly, the solutions of the surfactants were prepared separately: $4 \mathrm{~g}$ of P105 and $6 \mathrm{~g}$ of CrEL were dissolved in two $100-\mathrm{mL}$ volumetric flasks filled with deionized water $\left(\mathrm{DI}-\mathrm{H}_{2} \mathrm{O}\right)$. The two solutions were mixed at a $1: 1 \mathrm{v} / \mathrm{v}$ ratio. The mixture is then placed in a water bath at $45^{\circ} \mathrm{C}$, rotated at a speed of 80 for $20 \mathrm{~min}$, and then chilled on ice for $15 \mathrm{~min}$. The resultant mixed surfactant solution was refrigerated at $4-10^{\circ} \mathrm{C}$ until use.

\section{Preparation of nanoemulsions with Microfluidizer M110S (small scale)}

The preparation of the small-scale nanoemulsions (Table 1) is based on the already published formulations. ${ }^{6,31}$ Briefly, Miglyol $812 \mathrm{~N}$ and DiR dye stock solution were mixed and stirred at $350 \mathrm{rpm}$ for $15 \mathrm{~min}$, then PFPE oxide was added, and the solution was stirred for 15 min more. The surfactant mixture (P105/CrEL) was then combined and stirred for $15 \mathrm{~min}$ at the same speed. The final solution was then placed into an ice bath and sonicated for $1 \mathrm{~min}$, and then microfluidized on a precooled Microfluidizer M110S for 10 passes (operating liquid pressure $\sim 17,500 \mathrm{psi}$ ). For the celecoxibloaded nanoemulsion, the drug was predissolved in

Table 1. Nanoemulsion Scale-Up Formulations

\begin{tabular}{lrrrrrr}
\hline Components $^{\mathbf{a}}$ & A $^{\mathbf{b}}$ & $\mathbf{B}^{\mathbf{b}}$ & $\mathbf{C}$ & D & E & F \\
\hline Miglyol 812N & 4 & 4 & 20 & 20 & 74 & 74 \\
PFPE oxide & 4 & 4 & 20 & 20 & 74 & 74 \\
P105/CrEL 2:3 (5\% w/v) & 46 & 46 & 230 & 230 & 852 & 852 \\
Celecoxib (mg) & - & 10.3 & - & 51.6 & - & 190.9 \\
Total volume & 54 & 54 & 270 & 270 & 1,000 & 1,000 \\
\hline
\end{tabular}

${ }^{a}$ Values represent $\mathrm{mL}$ of liquid.

${ }^{\mathrm{b}}$ For the formulations A and B, DiR dye was incorporated. 
Miglyol $812 \mathrm{~N}$ overnight and the DiR dye stock solution was prepared in ethanol $(2 \mathrm{mM})$.

\section{Preparation of nanoemulsions with Microfluidizer}

M-110EH-30 (medium and large scale)

For the scale-up experiments (Table 1), we used a high shear fluid processor M-110EH-30. More specifically, for the medium scale $(270 \mathrm{~mL})$, Miglyol $812 \mathrm{~N}$ and PFPE oxide were mixed and stirred at $350 \mathrm{rpm}$ for $15 \mathrm{~min}$, the surfactant mixture (P105/CrEL) was added, and the solution was stirred for $15 \mathrm{~min}$ more at the same speed while it was placed into an ice bath. The pre-emulsification solution was sonicated for $2 \mathrm{~min}$ while stirring at $4^{\circ} \mathrm{C}$. Afterwards, the solution was microfluidized on a precooled Microfluidizer $\mathrm{M}$ 110EH-30 for five passes (operating liquid pressure $\sim 15,000 \mathrm{psi})$. For the large scale $(1,000 \mathrm{~mL})$, Miglyol $812 \mathrm{~N}$ and PFPE oxide were mixed and stirred at $350 \mathrm{rpm}$ for $15 \mathrm{~min}$, the surfactant mixture (P105/ CrEL) was added, and the solution was stirred at higher speed $(600 \mathrm{rpm})$ for $30 \mathrm{~min}$ in an ice bath. The preemulsification solution was sonicated for $5 \mathrm{~min}$ while stirring at $4^{\circ} \mathrm{C}$. Afterwards, the solution was microfluidized on a precooled Microfluidizer M-110EH-30 for three passes (operating liquid pressure $\sim 15,000 \mathrm{psi}$ ). For all the experiments, temperature was controlled for each step and celecoxib was predissolved in Miglyol $812 \mathrm{~N}$ overnight.

\section{DLS measurements for nanoemulsions}

Dynamic light scattering (DLS) of all nanoemulsion samples collected in stability studies was performed as earlier reported. ${ }^{6,31}$ Nanoemulsion samples were prepared by dilution in deionized water or other media at $1: 40 \mathrm{v} / \mathrm{v}^{6}$ All samples were analyzed at $25^{\circ} \mathrm{C}$ with a scattering angle of $173^{\circ}$ on Zetasizer Nano ZS (Malvern Instruments).

\section{Stability assessment under filtration}

and centrifugation

We determined the size distribution of the nanoemulsion droplets before and after filtration. Samples (10 mL volume) of undiluted nanoemulsions were filtered using $0.22-\mu \mathrm{m}$ syringe filters (Millex ${ }^{\circledR}-\mathrm{GS}$, Merck Millipore Ltd., MF-Millipore ${ }^{\mathrm{TM}}$ membrane). DLS measurements were taken after the nanoemulsion samples were diluted in water $(1: 40 \mathrm{v} / \mathrm{v})$ and allowed to equilibrate at room temperature for at least $30 \mathrm{~min}$ before each measurement. All DLS measurements were done at room temperature. ${ }^{6} \mathrm{We}$ also determined the size distribution of the nanoemulsion droplets before and after centrifugation. The nanoemulsions were diluted $(1: 40 \mathrm{v} / \mathrm{v})$ in water, serum-free DMEM, $10 \%$ FBS DMEM, or $20 \%$ FBS DMEM. Diluted nanoemulsions were centrifuged using Centrifuge 5804R (VWR; Eppendorf AG) at 1,100 rpm for $10 \mathrm{~min}$. The samples were then transferred to DLS cuvettes, and measurements were taken after equilibrating at room temperature for at least $30 \mathrm{~min}$ before each measurement. All measurements were done at room temperature. ${ }^{6}$ Samples were analyzed at $25^{\circ} \mathrm{C}$ with a scattering angle of $173^{\circ}$.

\section{Stability assessment in cell culture media}

Furthermore, the colloidal stability of nanoemulsions in biological media was also evaluated by measuring the droplet diameter. More specifically, for these stress studies, nanoemulsions were incubated at $37^{\circ} \mathrm{C}(1: 40 \mathrm{v} /$ $\mathrm{v}$ ) in deionized water and cell culture medium (serumfree DMEM, 10\% and 20\% FBS DMEM) for up to $72 \mathrm{~h}$. Undiluted samples were measured for droplet size, polydispersity, and zeta potential at $25^{\circ} \mathrm{C}$ with a scattering angle of $173^{\circ}$ on Zetasizer Nano.

\section{$\mathrm{pH}$ measurements}

The $\mathrm{pH}$ values of the nanoemulsions were measured at ambient temperature using a double-junction, glass body refillable $\mathrm{pH}$ electrode (Oakton) attached to an Oakton pH meter 1100 series. Before taking any measurements, the $\mathrm{pH}$ meter was calibrated using two standard buffers from Fisher Scientific at pH 4.00 (certified $\mathrm{pH} 3.99-4.01$ at $25^{\circ} \mathrm{C}$ ) and 7.00 (certified $\mathrm{pH}$ 6.99-7.01 at $\left.25^{\circ} \mathrm{C}\right)$.

\section{Cell culture}

Cell viability. Cell viability was assessed using CellTiterGlo ${ }^{\circledR}$ luminescence assay as reported earlier. ${ }^{6}$ Briefly, mouse macrophages (Raw 264.7) were seeded in a 96-well plate at 10,000 cells per well. After overnight incubation $(18-20 \mathrm{~h})$ at $37^{\circ} \mathrm{C}$ and $5 \%$ of $\mathrm{CO}_{2}$, culture media were removed and adherent cells were exposed for $24 \mathrm{~h}$ to nanoemulsions $\mathrm{A}, \mathrm{B}, \mathrm{C}, \mathrm{D}, \mathrm{E}$, and F (prediluted in fresh, warm complete media) at different nanoemulsion dilutions $(0-79.4 \mu \mathrm{L}$ of nanoemulsion/ $1 \mathrm{~mL}$ of media). Upon completed incubation, $40 \mu \mathrm{L}$ of CellTiter-Glo reagent was added to each well. Plates were covered with aluminum foil to protect samples from light and mixed on Lab Doctor ${ }^{\mathrm{TM}}$ Orbital Shaker at the speed of 70 for $20 \mathrm{~min}$. Luminescence was 
recorded on a microplate reader (1420 Multilabel Counter, Victor ${ }^{3 \mathrm{TM}}$; Perkin Elmer).

$\mathrm{PGE}_{2}$ assay. To investigate the in vitro therapeutic efficiency of the drug carrier for the scale-up nanoemulsions, the effect of these nanoemulsions on prostaglandin $\mathrm{E}_{2}\left(\mathrm{PGE}_{2}\right)$ production by macrophages was assessed by comparing the effect on $\mathrm{PGE}_{2}$ production with free drug (celecoxib solution in DMSO). RAW 246 cells were seeded in a six-well plate at 0.8 million cells per well and incubated overnight. We expose the cells with nanoemulsions $\mathrm{E}$ and $\mathrm{F}$ at $1.4 \mathrm{mg} /$ $\mathrm{mL}$ PFPE concentration $(9.28 \mu \mathrm{M}$ celecoxib), free drug dissolved in DMSO $(9.28 \mu \mathrm{M})$, and DMSO for $24 \mathrm{~h}$. Fresh media were added to unexposed cells. After overnight incubation, all cells were washed with DPBS $(2 \times)$. Bacterial toxin lipopolysaccharide (LPS) at $500 \mathrm{ng} / \mathrm{mL}$ in the full culture media was added to each well $(2 \mathrm{~mL}$ in each well) with exposed and unexposed cells incubating for $4 \mathrm{~h}$. Unexposed cells treated with LPS were designed as control, and unexposed cells without LPS stimulation were designed as untreated. After $4 \mathrm{~h}$ of incubation, supernatant was collected and analyzed using the commercially available $\mathrm{PGE}_{2}$ ELISA kit. Samples were analyzed at two different dilutions (1:5 and 1:10) and triplicates of each dilution were used. Assessment of $\mathrm{PGE}_{2}$ production in the supernatant and data analysis were performed according to the manufacturer's instructions. ${ }^{6}$

\section{Results and Discussion}

In this report, we focused on demonstrating the feasibility of producing theranostic nanoemulsions on scale. We also explored the effects of microfluidization instrumentation used on the nanoemulsion product quality. Nanoemulsions, produced on three scales, were evaluated by a combination of measurements, including DLS for size distribution and zeta potential and $\mathrm{pH}$ measurements for stability. Stability assessments were performed upon storage for up to 90 days and in biologically representative media for up to $72 \mathrm{~h}$. We also evaluated nanoemulsions for their effects on model inflammatory cells in vitro. Cell toxicity was tested with nanoemulsion effect on COX-2 in activated macrophages. The presented data indicate a high level of processing robustness and stability of produced nanoemulsions.

Reported theranostic nanoemulsions are produced by microfluidization at three different scales, small $(54 \mathrm{~mL})$, medium $(270 \mathrm{~mL})$, and large $(1,000 \mathrm{~mL})$ (Table 1). Our earlier reported methods for PFC nanoemulsion preparation were adapted to accommodate
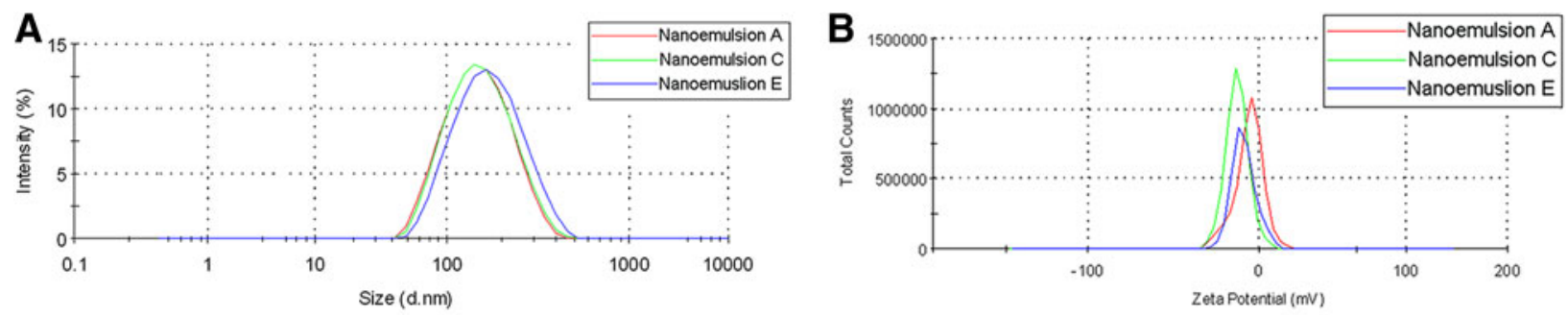

C
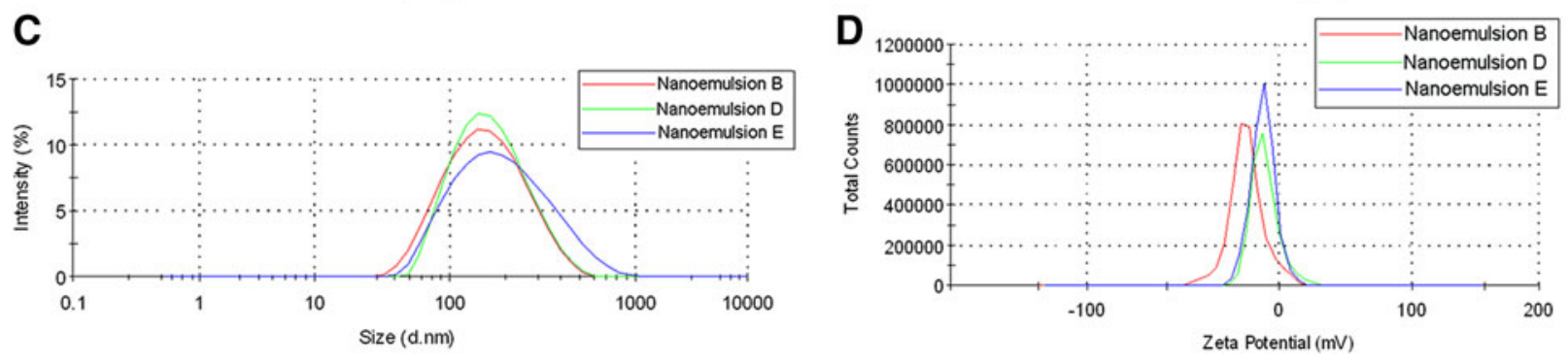

FIG. 1. Size distribution and zeta potential comparison between small ( $54 \mathrm{~mL}$, nanoemulsions $A$ and $B)$, medium (270 mL, nanoemulsions $C$ and $D)$ and large scale $(1,000 \mathrm{~mL}$, nanoemulsions $E$ and $F)$. (A) Size distribution and (B) zeta potential comparison for nanoemulsions without the drug; (C) Size distribution and (D) Zeta potential comparison for nanoemulsions with the drug. 

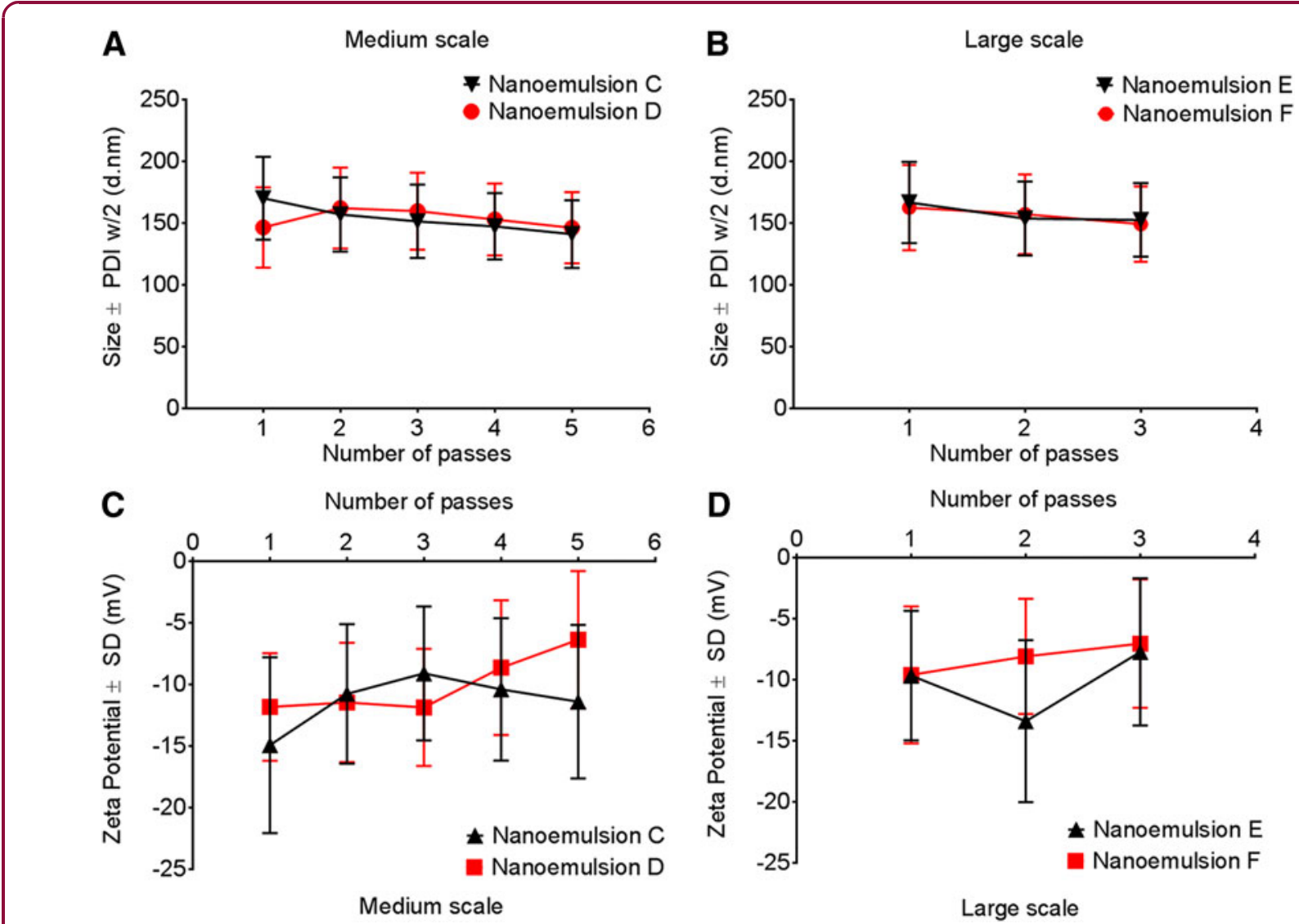

FIG. 2. Effect of number of passes on size, polydispersity, and zeta potential for medium- and large-scale nanoemulsions. (A) Size and polydispersity of nanoemulsions without drug, nanoemulsion $C$, and with drug, nanoemulsion D, on medium scale $270 \mathrm{~mL}$; (B) Size and polydispersity of nanoemulsions without drug, nanoemulsion $E$, and with drug, nanoemulsion $F$, on large scale 1,000 mL; (C) Zeta potential for medium-scale nanoemulsions $C$ and $D$; (D) Zeta potential for medium-scale nanoemulsions $E$ and $F$.

the increase in processing volume and change of instrumentation. $^{6,31}$ The small-scale nanoemulsions were processed on the Microfluidizer M110S, and the medium- and large-scale nanoemulsions were processed on the Microfluidizer M-110EH-30. Our DLS measurements indicated that the nanoemulsion particle size distribution and zeta potential distribution were not significantly affected by the nanoemulsification scale or instrument used (Fig. 1). Furthermore, the number of passes was progressively decreased as the scale was increased to prevent product heating. ${ }^{32}$ The nanoemulsion temperature at the small scale in between passes was $\sim 8-10^{\circ} \mathrm{C}$, but when the production was scaled up, the temperature was elevated to $\sim 23-24^{\circ} \mathrm{C}$. This temperature increase did not affect the quality of the final products, as indicated in Figure 1. No change in size or polydispersity index (PDI) was observed by decreasing the number of passes between small (10 passes), medium (5 passes), and large scale (3 passes) (Fig. 2). These observations are significant because they indicate robustness of the method and allow for savings in processing time, which further increases applicability to theranostics loaded with temperature or shear-sensitive drugs and/or imaging moieties. These results strengthen the argument for decreasing the number of passes during the manufacturing process, which can lead to decreased production costs.

Furthermore, our measurements indicated that the presence of the drug in produced nanoemulsions did not affect size, zeta potential, or $\mathrm{pH}$ (Fig. 3) by production of three different scales and using two types of microfluidizers. Applicability of theranostic nanoemulsions 

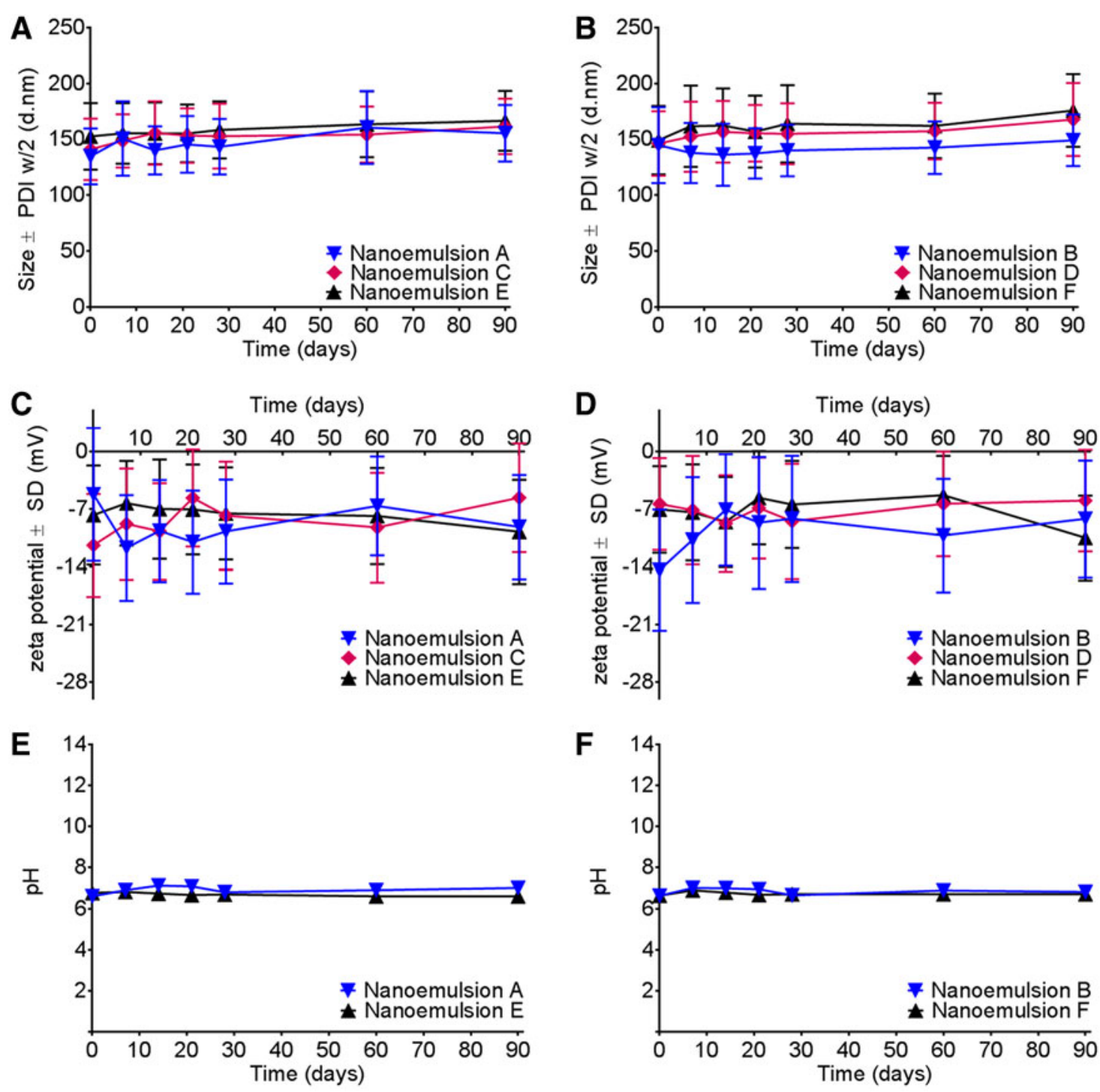

FIG. 3. Nanoemulsion stability evaluations. Effects of scale on particle size and zeta potential measurements and $\mathrm{pH}$ upon storage at $4^{\circ} \mathrm{C}$ for 90 days. (A) Size measurements of nanoemulsions ( $\mathrm{A}, \mathrm{C}$, and $\mathrm{E}$ ) without drug; (B) Size measurements of drug-loaded nanoemulsions (B, D, and F); (C) Zeta potential measurements of nanoemulsions ( $A, C$, and $E$ ) without drug; (D) Zeta potential measurements of drug-loaded nanoemulsions ( $B$, $\mathrm{D}$, and $\mathrm{F}$ ); (E) $\mathrm{pH}$ measurements of nanoemulsions $\mathrm{A}$ and $\mathrm{E}$ without a drug; (F) $\mathrm{pH}$ measurements of drugloaded nanoemulsions $B$ and $F$.

in advanced preclinical testing and clinical trials in the future relies on their quality. To evaluate their shelf life, we tested all produced nanoemulsions for droplet size, polydispersity, zeta potential, and $\mathrm{pH}$ at storage conditions $\left(4^{\circ} \mathrm{C}\right)$ for 90 days. Figure $3 \mathrm{~A}$ and $\mathrm{B}$ shows no significant change in droplet size after 90 days of follow-up for nanoemulsions with and without a drug at all levels of the scale (small, medium, and large). Zeta potential was also maintained during the 90-day followup at around $-7 \mathrm{mV}$, which further supports the stability of nanoemulsions (Fig. 3C, D). Furthermore, the nanoemulsion $\mathrm{pH}$ remained stable at $\sim 6.8$ (Fig. 3E, F). Once nanoemulsions are used in vivo, they come into contact with complex biological fluids. To model 
these stressors, we evaluated their colloidal stability in model biological media (FBS-containing cell culture media) at an elevated temperature $\left(37^{\circ} \mathrm{C}\right)$ for $72 \mathrm{~h}$. No significant changes in size and polydispersity were observed upon $72 \mathrm{~h}$ of incubation. This suggests that all nanoemulsions (small, medium, large scale) with or without the drug are not affected by the presence of protein, salts, or nutrients (Fig. 4A-F). To further investigate nanoemulsion stability under mechanical stress and the potential impact of the scale of production on nanoemulsion quality, we performed centrifugation and filtration stability tests. It was found that upon
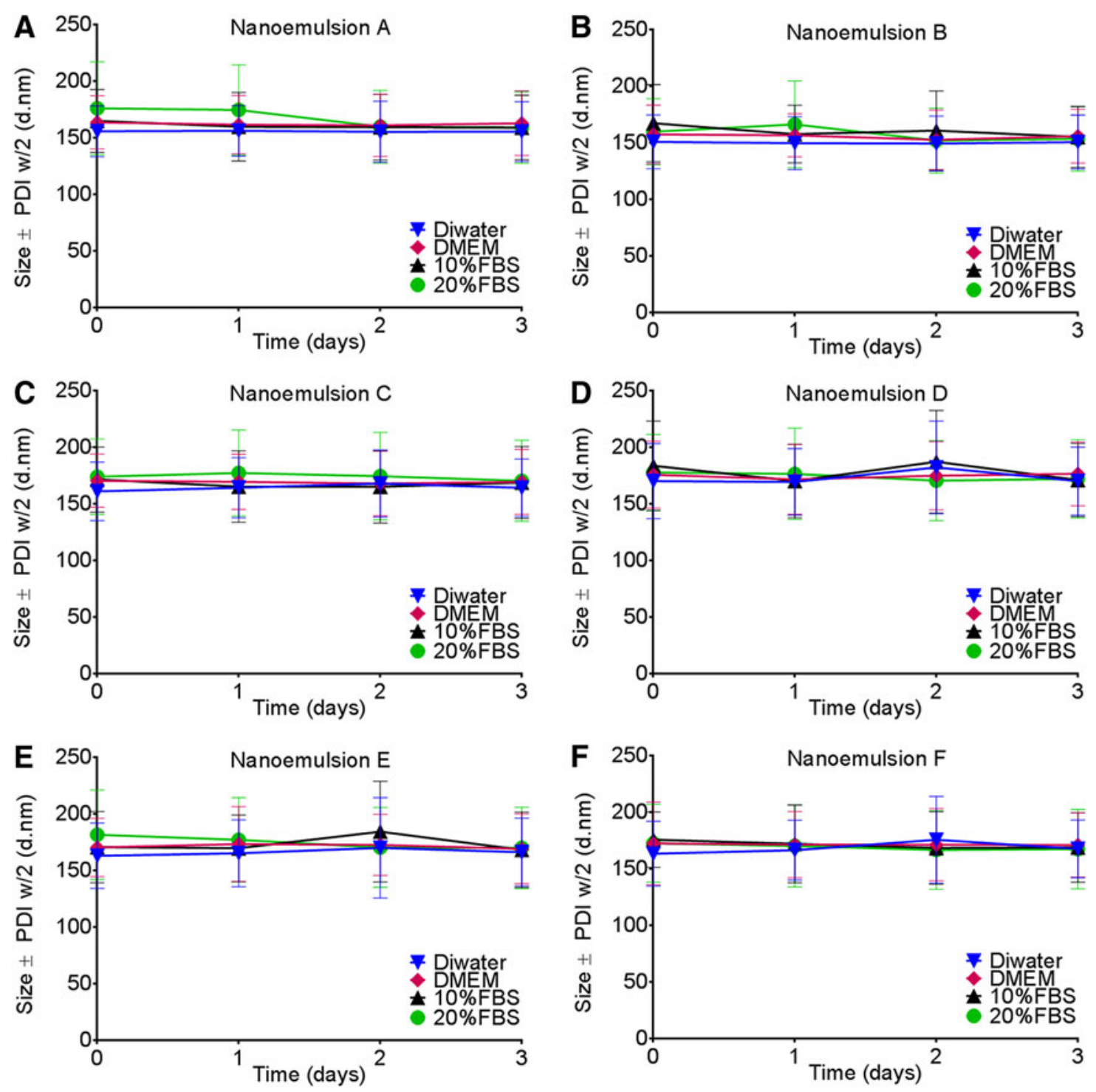

FIG. 4. Nanoemulsion stability in biological media measured by dynamic light scattering (DLS) for small-scale nanoemulsions with/without a drug (A, B), medium scale (C, D), and large scale (E, F). Nanoemulsions were diluted at $1 / 40(\mathrm{v} / \mathrm{v})$ in deionized water, serum-free Dulbecco's modified Eagle's medium (DMEM) cell culture media, 10\% fetal bovine serum (FBS), and 20\% FBS DMEM and incubated for up to $72 \mathrm{~h}$ at body temperature $\left(37^{\circ} \mathrm{C}\right)$. Samples were tested by DLS for size and polydispersity at times $0,24,48$, and $72 \mathrm{~h}$. Data represent average droplet size (Z-average) with standard deviation as half of polydispersity width. Incubation samples were measured by DLS, undiluted. 

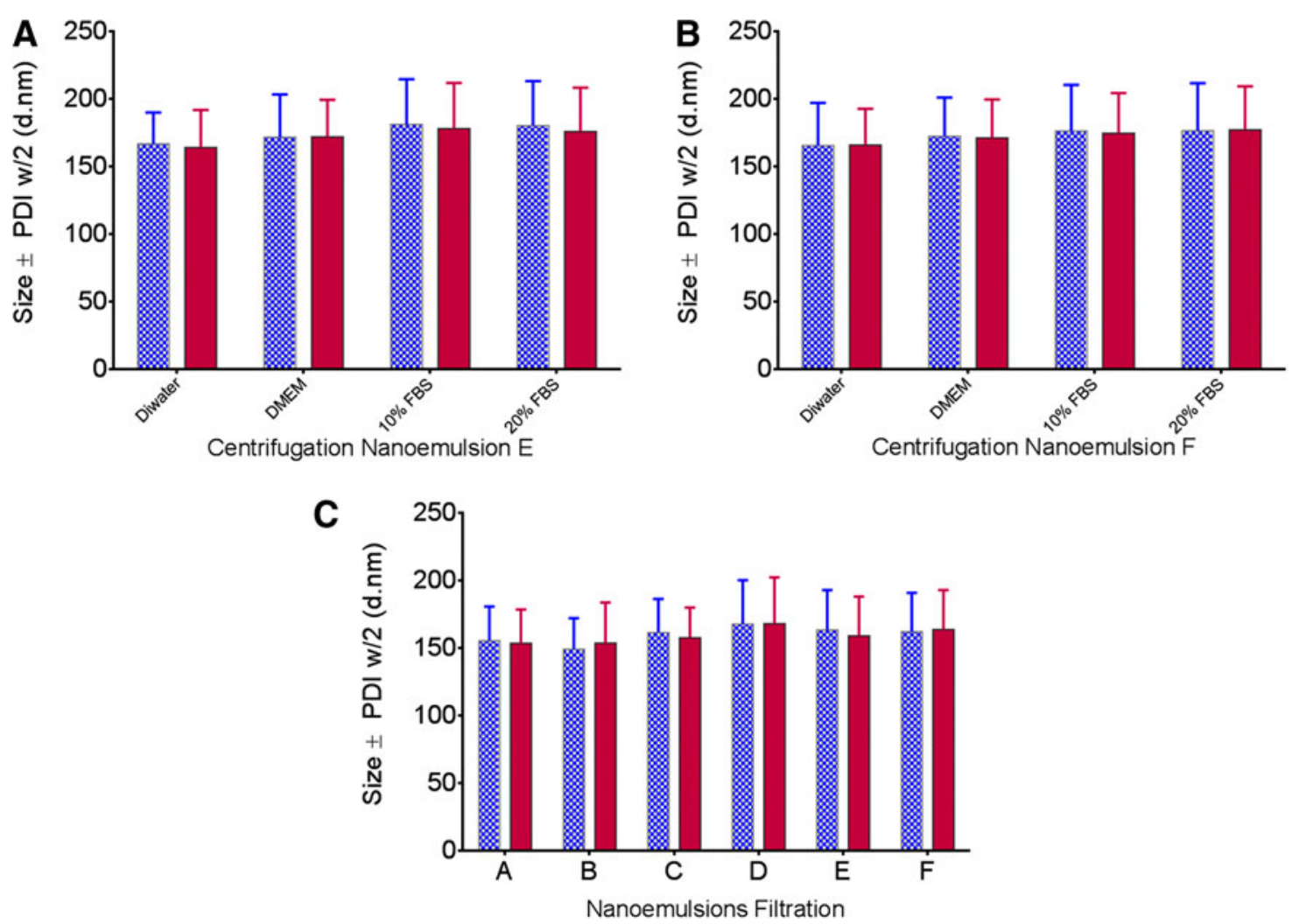

FIG. 5. Centrifugation stability of large-scale nanoemulsions $E(\mathbf{A})$ and $F(B)$; filtration stability for nanoemulsion A, B, C, D, E, and F (C). In all plots, standard deviation represents polydispersity width half (PDIw/ 2), indicating the size distribution for each nanoemulsion under testing conditions. Blue open bars represent measurements before the stress induction, and solid red bars are measurements after the stress was applied (centrifugation or filtration).

centrifugation at $1,100 \mathrm{rpm}$ for $5 \mathrm{~min}$ at room temperature and in different media (water, serum-free, and serum-containing cell culture media), the largest scale nanoemulsions ( $\mathrm{E}$ and $\mathrm{F}$ ) showed no significant changes in size and polydispersity (Fig. 5A, B). When the nanoemulsions were tested against filtration through a $0.22-$ $\mu \mathrm{m}$ filter, all nanoemulsions (small, medium, large scale) prepared with or without the drug showed no changes in size and polydispersity (Fig. 5C). These data suggest that all nanoemulsions reported here at three levels of scale remain highly stable when exposed to stressors during storage and use, as we earlier reported for small-scale preparations. ${ }^{6,31}$

We also investigated scale-up nanoemulsions in cellbased assays. To evaluate their effects on cell viability, we used the mouse macrophage cell line (RAW 264.7). Upon overnight incubation at increasing con- centration (up to $80 \mu \mathrm{L}$ loading volume of nanoemulsion per $1 \mathrm{~mL}$ of media), we found no significant change in cell viability using CellTiter-Glo ${ }^{\circledR}$ Luminescent Cell Viability assay. As a control, we used free drug (celecoxib [Coxb] dissolved in DMSO) at the same concentration levels as in the nanoemulsions, DMSO only as a negative control, and doxorubicin as a positive control. Figure 6 summarizes cell toxicity assays. Finally, we tested the effects of large-scale nanoemulsion on COX-2 enzyme activity in macrophages, following the same experimental set-up as reported earlier. ${ }^{6}$ When exposed to LPS, macrophages upregulate the COX-2 enzyme, which leads to increased production of $\mathrm{PGE}_{2}$. As in the previous study, we show here that exposure to celecoxib-loaded nanoemulsion dramatically reduces release of $\mathrm{PGE}_{2}$ from RAW 264.7 cells. Figure 7A illustrates the proposed mechanism of 

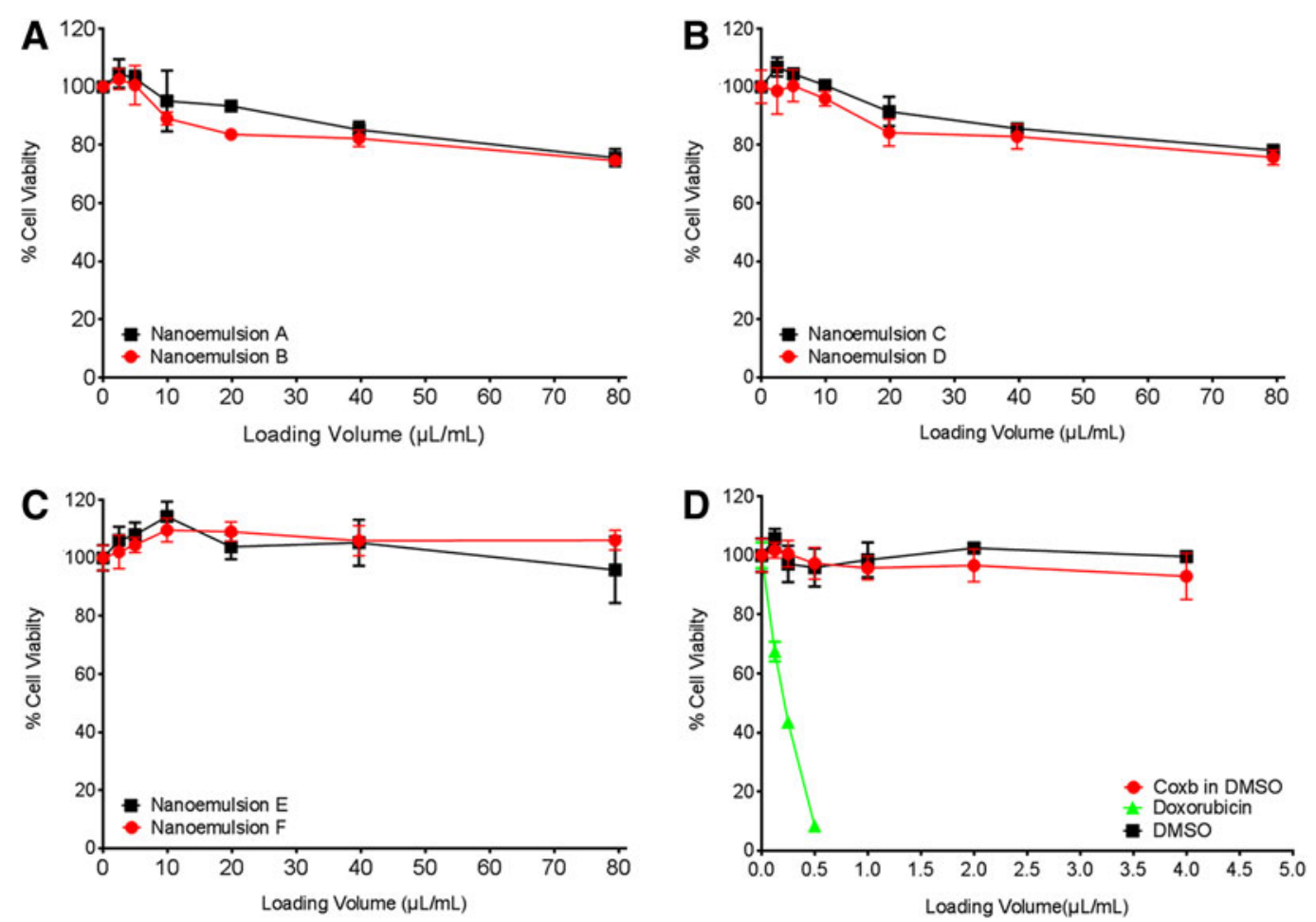

FIG. 6. Cell viability testing for nanoemulsions produced at three levels of scale (small, medium, and large). (A) Cell viability percentage of small-scale nanoemulsions A and B; (B) Cell viability percentage of mediumscale nanoemulsions C and D; (C) Cell viability percentage of large-scale nanoemulsions $E$ and F; (D) Control cell viability test with free drug (celecoxib in DMSO) at equivalent concentrations to nanoemulsion cell exposures, DMSO (vehicle), and doxorubicin (positive control). All data represent an average $\pm S D(N=3)$.
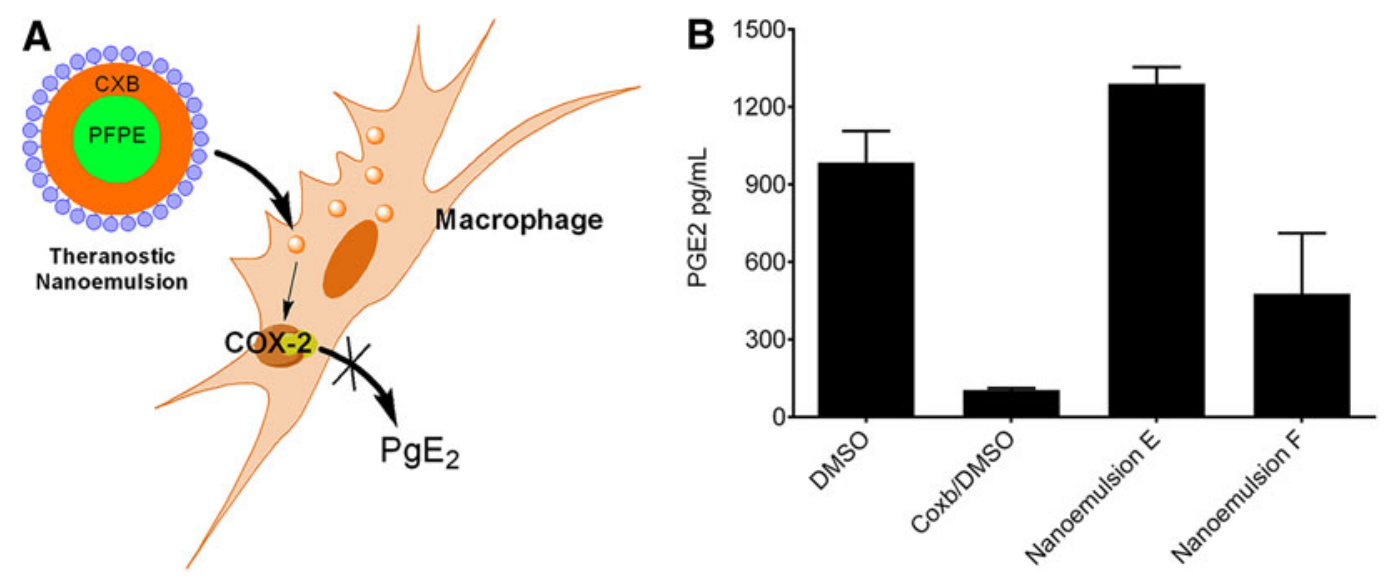

FIG. 7. (A) Celecoxib-loaded theranostic nanoemulsion inhibits COX-2 enzyme in lipopolysaccharide (LPS)activated macrophages; (B) prostaglandin $\mathrm{E}_{2}\left(\mathrm{PGE}_{2}\right)$ release inhibition from LPS-activated macrophages exposed to celecoxib-loaded nanoemulsion $\mathrm{F}$ and drug-free nanoemulsion $\mathrm{E}$. Free drug (celecoxib, Coxb) and DMSO were used as controls. Data represent the average from three independent measurements (mean \pm SD). 
theranostic nanoemulsions on the COX-2 enzyme. Largest scale $(1,000 \mathrm{~mL})$ drug-loaded nanoemulsion demonstrated significant inhibition of $\mathrm{PGE}_{2}$ release compared with vehicle drug-free nanoemulsions (Fig. 7B). Celecoxib in DMSO was used as the positive control. This result demonstrates that we successfully scaled up a theranostic nanoemulsion and maintained its biological safety (Fig. 6) efficacy (Fig. 7).

\section{Conclusion}

In this study, we report for the first time the successful scale of theranostic nanoemulsions. Processing changes such as instrumentation used and processing temperature did not affect the final product quality in respect to size, polydispersity, zeta potential, or $\mathrm{pH}$. Long-term follow-up upon storage shows no change in stability for all nanoemulsions produced regardless of the scale and presence of drug for up to 90 days. Furthermore, cell assays indicated comparable cell viability profiles between nanoemulsions and with earlier reported data. When large-scale $(1 \mathrm{~L})$ nanoemulsion was tested for anti-inflammatory effects in a model cell line (mouse macrophages, RAW 264.7), it showed, as expected, inhibition of the cyclooxygenase 2 (COX-2) enzyme leading to reduced $\mathrm{PGE}_{2}$ release. Furthermore, we also show high stability of all nanoemulsions produced when subjected to a variety of stress tests, such as exposure to biologically relevant media, filtration, and centrifugation. These results strongly suggest that theranostic nanosystems if designed with the scale in mind can become viable clinical candidates. We hope this report spurs new investigations and pharmaceutical formulations in the field of theranostic nanomedicine. Producing nanomedicines on a sufficient scale and with maintained quality leads to high quality of pre-clinical testing by removing batch-to-batch variability.

\section{Acknowledgments}

The authors acknowledge assistance by Microfluidics Corporation (Westwood, MA) who generously provided Microfluidizer M-110EH-30 to support this study. C.B. and J.M.J. are supported by the Duquesne University Start-up Research Funds. L.L. was supported by a summer stipend from the Chronic Pain Research Consortium (CPRC), funded by Provost's Interdisciplinary Research Consortia Grant at Duquesne University.

\section{Author Disclosure Statement}

No competing financial interests exist.

\section{References}

1. Lammers T, et al. Theranostic nanomedicine. Acc Chem Res. 2011;44:1029-1038.

2. Pan D. Theranostic nanomedicine with functional nanoarchitecture. Mol Pharmacol. 2013;10:781-782.

3. Prabhu P, Patravale V. The upcoming field of theranostic nanomedicine: an overview. J Biomed Nanotechnol. 2012;8:859-882.

4. Rizzo LY, et al. Recent progress in nanomedicine: therapeutic, diagnostic and theranostic applications. Curr Opin Biotechnol. 2013;24:1159-1166.

5. Ahrens ET, Bulte JW. Tracking immune cells in vivo using magnetic resonance imaging. Nat Rev Immunol. 2013;13:755-763.

6. Patel SK, et al. Cyclooxgenase-2 inhibiting perfluoropoly (ethylene glycol) ether theranostic nanoemulsions-in vitro study. PLoS One. 2013;8:e55802.

7. McClements DJ. Nanoemulsions versus microemulsions: terminology, differences, and similarities. Soft Matter. 2012;8:1719-1729.

8. Shakeel F, et al. Nanoemulsions as potential vehicles for transdermal and dermal delivery of hydrophobic compounds: an overview. Expert Opin Drug Deliv. 2012;9:953-974.

9. Kotta $S$, et al. Exploring oral nanoemulsions for bioavailability enhancement of poorly water-soluble drugs. Expert Opin Drug Deliv. 2012;9:585598.

10. McClements DJ, Rao J. Food-grade nanoemulsions: formulation, fabrication, properties, performance, biological fate, and potential toxicity. Crit Rev Food Sci Nutr. 2011;51:285-330.

11. Rajpoot $P$, Pathak K, Bali V. Therapeutic applications of nanoemulsion based drug delivery systems: a review of patents in last two decades. Recent Pat Drug Deliv Formul. 2011;5:163-172.

12. Sarker DK. Engineering of nanoemulsions for drug delivery. Curr Drug Deliv. 2005;2:297-310.

13. Mitri $K$, et al. Scale-up of nanoemulsion produced by emulsification and solvent diffusion. J Pharm Sci. 2012;101:4240-4247.

14. Muller RH, Harden D, Keck CM. Development of industrially feasible concentrated $30 \%$ and $40 \%$ nanoemulsions for intravenous drug delivery. Drug Dev Ind Pharm. 2012;38:420-430.

15. Constantinides PP, Chaubal MV, Shorr R. Advances in lipid nanodispersions for parenteral drug delivery and targeting. Adv Drug Deliv Rev. 2008;60:757-767.

16. Maali A, Mosavian MTH. Preparation and application of nanoemulsions in the last decade (2000-2010). J Disper Sci Technol. 2013;34:92-105.

17. Ahrens ET, Zhong J. In vivo MRI cell tracking using perfluorocarbon probes and fluorine-19 detection. NMR Biomed. 2013;26:860-871.

18. Hitchens TK, et al. 19F MRI detection of acute allograft rejection with in vivo perfluorocarbon labeling of immune cells. Magn Reson Med. 2011;65:1144-1153.

19. Kadayakkara DK, et al. In vivo observation of intracellular oximetry in perfluorocarbon-labeled glioma cells and chemotherapeutic response in the CNS using fluorine-19 MRI. Magn Reson Med. 2010;64:1252-1259.

20. Rapoport N, et al. Ultrasound-mediated tumor imaging and nanotherapy using drug loaded, block copolymer stabilized perfluorocarbon nanoemulsions. J Control Release. 2011;153:4-15.

21. Akers WJ, et al. Noninvasive photoacoustic and fluorescence sentinel lymph node identification using dye-loaded perfluorocarbon nanoparticles. ACS Nano. 2011;5:173-182.

22. Fraker $C A$, et al. Optimization of perfluoro nano-scale emulsions: the importance of particle size for enhanced oxygen transfer in biomedical applications. Colloids Surf B Biointerfaces. 2012;98:26-35.

23. Spahn DR, Kocian R. Artificial O2 carriers: status in 2005. Curr Pharm Des. 2005;11:4099-4114.

24. Srinivas $M$, et al. In vivo cytometry of antigen-specific t cells using $19 \mathrm{~F}$ MRI. Magn Reson Med. 2009;62:747-753.

25. Stoll G, et al. Visualization of inflammation using (19) F-magnetic resonance imaging and perfluorocarbons. Wiley Interdiscip Rev Nanomed Nanobiotechnol. 2012;4:438-447.

26. Balducci $A$, et al. A novel probe for the non-invasive detection of tumorassociated inflammation. Oncoimmunology. 2013;2:e23034.

27. Kadayakkara DK, et al. Inflammation driven by overexpression of the hypoglycosylated abnormal mucin 1 (MUC1) links inflammatory bowel disease and pancreatitis. Pancreas. 2010;39:510-515.

28. Weise $G$, et al. In vivo imaging of inflammation in the peripheral nervous system by (19)F MRI. Exp Neurol. 2011;229:494-501. 
29. Dewitte $\mathrm{H}$, et al. Design and evaluation of theranostic perfluorocarbon particles for simultaneous antigen-loading and (19)F-MRI tracking of dendritic cells. J Control Release. 2013;169:141-149.

30. Lanza GM. Targeted antiproliferative drug delivery to vascular smooth muscle cells with a magnetic resonance imaging nanoparticle contrast agent: implications for rational therapy of restenosis. Circulation. 2002;106:2842-2847.

31. Janjic JM, et al. Perfluorocarbon nanoemulsions with fluorescent, colloidal and magnetic properties. Biomaterials. 2014;35:4958-4968.

32. Pinnamaneni S, Das NG, Das SK. Comparison of oil-in-water emulsions manufactured by microfluidization and homogenization. Pharmazie. 2003;58:554-558.

Cite this article as: Liu L, Bagia C, Janjic JM (2015) The first scale-up production of theranostic nanoemulsions. BioResearch Open Access 4:1, 218-228, DOI 10.1089/biores.2014.0030.

\section{Abbreviations Used}

DCs $=$ dentritic cells

$\mathrm{DLS}=$ dynamic light scattering

LPS $=$ lipopolysaccharide

$\mathrm{PFC}=$ perfluorocarbon

$\mathrm{PGE}_{2}=$ prostaglandin $\mathrm{E}_{2}$

\section{Publish in BioResearch Open Access}

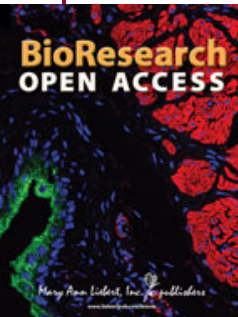

- Broad coverage of biomedical research - Immediate, unrestricted online access

- Rigorous peer review

- Compliance with open access mandates

- Authors retain copyright

- Highly indexed

- Targeted email marketing

liebertpub.com/biores 